\title{
An In-Depth Study in TCA Cycles, OPA1, S6K1, ATPase, TLR4, MHC-class-I, GCs, and IFNs Bio-Synthesis, and Their Roles of Deficiency in Diabetes, Asthma, Cancer, etc and the NAD Roles in Their Activities
}

Research Article

Volume 1 Issue 2 - 2021

\begin{abstract}
Author Details
Ashraf Marzouk El Tantawi*

Biomedical molecular studies, Egypt

*Corresponding author

Ashraf Marzouk El Tantawi, Biomedical molecular studies, Egypt
\end{abstract}

Article History

Received: September 20, $2021 \quad$ Accepted: October14, 2021 Published: October 19, 2021

\begin{abstract}
The roles of S6K1 is regulating ATPase, and GTPase synthesis, and consequently the endocytic proliferations including endocytic soluble MHC class II synthesis which regulate both SIRP $\alpha 1$ and TLR4 synthesis, where diabetes reflect deficiency in Ser amino acids that reflect deficiency in pyrimidines synthesis consequently deficiency in Estrogen and reflect increasing in androgen synthesis with increasing in consuming in purines (A\&G) that lead to decreasing in anabolic processes which depends on presence of adenosine and guanosine stored in ribosomes. OPA1-synthetase enzymes are necessary for producing amino-acyl-CoA-synthetase (gamma-subunits) which regulate glucocorticoids synthesis, and Interferons isoforms productions, for reactivating macrophages, and MHC class-I which regulate the endocytic soluble MHC class II synthesis which regulate both SIRP $\alpha 1$ and TLR4 productions for cellular proliferation. S6K1 promote ATPase synthesis for regulating Interferons (IFNs) isoforms synthesis and regulated by pyrimidine-kinases (PS/T Adenosine Kinase \& PS/T-Guanosine Kinase) productions. Asthma is characterized by Prediabetes and diabetes, that the reasons of causing the two diseases (Asthma \& diabetes) are the deficiency in Ser amino acids and consequently deficiency in pyrimidine kinases productions lead to increasing in Androgen production but deficiency in Estrogen biosynthesis. Estrogen formed from Ser/ Thr mTOR FOX phosphorylations signaling pathways for glucocorticoids synthesis regulated and modified by OPA1 enzymes effects for producing acyl-CoA-glucocorticoids isoforms, but the feedback of GC to produce estrogen will be upon ATPase \& synthetase enzymes on GCs isoforms followed by Ser/Thr phosphorylation signaling pathway which will promote both Estrogen, protein kinases, and S6K1 production.

The Purpose of this study: The roles of S6K1 is regulating ATPase, and GTPase synthesis, and consequently regulate endocytic soluble MHC class II synthesis which regulate SIRP $\alpha 1$ synthesis for promoting TLR4 synthesis. Diabetes reflect deficiency in pyrimidines nucleotides and consequently deficiency in Estrogen with increasing in androgen synthesis that after period of time will increase the consuming of purines (A\&G) in ATPase synthesis that lead to decreasing in anabolic processes and proliferations too. OPA1-synthetase enzymes are so necessary for producing amino-acyl-CoA-synthstase (gamma-subunits) and signals, where gamma-subunits are the basics for regulating glucocorticoids synthesis, and Interferons isoforms productions, for reactivating macrophages, through MHC class-I synthesis which migrate to nucleated cells for endocytic soluble MHC class II synthesis which promote both SIRP $\alpha 1$ and TLR4 synthesis upon phospholipase effects on Beta-subunit "fatty-acyl-CoA-synthase subunits". S6K1 consider as protected basic subunits for Interferons (IFNs) isoforms synthesis regulated by pyrimidine-kinases. Asthma is characterized by Prediabetes and diabetes, that reasons of causing the two disease (Asthma \& diabetes) are same which is due to deficiency in Ser amino acids and deficiency in pyrimidine synthesis which regulate by synthetase enzymes. Estrogen formed from Ser/Thr- FOX signaling pathways for glucocorticoids synthesis (and for IFNs synthesis) Upon OPA1 enzymes effects on estrogen.
\end{abstract}




\section{Material:}

FOX genes,

S6K1, ATPase and GTPase

OPA1 enzymes,

MTORC1, PS/T-Thymine-K, PS/T-Adenosene-k, PS/T-Guanosine-kinases

Fatty-acyl-CoA-synthetase,

Fatty-acyl-CoA-synthase,

Fatty-acyl-CoA-phospholipase

N'A, K, ATPase

Renal proximal tubule epithelia

Beta-cells and beta chains

CD8 T cells

IFN-gamma, IFN-beta, and IFN-alpha

Diabetes and asthma studies

MHC Class-I, MHC class II

Signal proteins

Tyrosine metabolic pathways

Arg metabolism and proline synthesis

Mast cells

Nicotinamide adenine dinucleotide, oxidized form (NAD+).

T-cells

B-cells

TGF-gamma, beta, alpha

TLR4

Myeloid cells

SIRP-gamma, SIRP-beta, SIRP- $\alpha$

Phosphatidic acid (PA)

\section{Introduction}

The purines and pyrimidines synthesis are the main basic units for rebuilding all of proper active subunits and active genes, where deficiency in some of necessary purines or pyrimidine will lead to symptoms of diseases. Mitochondrial TCA cycles are a regulated metabolite that related and depending on Ser/Thr FOX phosphorylation pathways functions, and on ATPase with COX activities on carbohydrate, on lipid, and on lipoprotein molecules for producing first long fatty acids chains and lipo-polysaccharides (LPS molecules) which will be oxidized and modified by OPA1 enzymes for producing three fatty-acyl-CoAs isoforms. The Ser/Thr phosphorylation signaling pathway firstly promote the productions of the four GPS of kinases (PS/TCk, PS/TTK, PS/TGk and PS/TAkinases), which will be oxidized and modified by mitochondrial enzymes (synthetase, synthase and phospholipase for producing only three fatty acyl-CoA isoforms (gamma, beta, then alpha upon synthetase, synthase, then phosphatase effects respectively).

The proper productions of PS/TG-kinases and PS/TA-kinase GPS depending on Ser/Thr FOX phosphorylation pathways, on availability of Ser and Thr amino acids, and on ATPase with COX enzymes for producing proper $\mathrm{S} 6 \mathrm{~K} 1$ peptides (which are formed from purines kinases mainly adenosine and guanosine protein kinases) which will be directed to ribosome for ATPase productions and for OPA1 repairs which regulated by GTPase. Mitochondrial OPA1 membrane regulate pyrimidine synthesis upon synthetase effects for producing gamma-subunits, where, the releasing of PS/TGk and PS/TAk are depending on the TCA cycles activities for producing purines kinases (PS/T-Adenosine kinase and PS/T-Guanosine kinases) which can be modified by OPA1 enzymes for producing acyl-CoAs rich of purines kinases, where TCA produce purines which will be used with pyrimidine (which produced from synthetase effects on LPS and on fatty long kinases proteins ) for necessary hydrophobic acids synthesis. The releasing of PS/T-Thymine -Kinase and PS/T-Cytosine -kinase chains (mTORC1) are regulating hydrophobic amino acids synthesis which can be modified by synthetase enzymes with purines which produced from TCA cycles for rebuilding the first gamma-subunits (upon synthetase effects) which promote the synthesis of Beta-subunit (upon synthase effect) and then alpha subunits upon phospholipase respectively. S6K1 synthesis and functions regulate tubulin acetylation (due to $\mathrm{S} 6 \mathrm{~K} 1$ is main regulator active peptides for ribosomal repairs and ATPase productions), hence $\mathrm{S} 6 \mathrm{~K} 1$ active subunits are contributing the Autophagic flux induced by different stress conditions and in different cells. Adenosine kinases (PS/T-Adenosine-Kinases) is one of the two substrates (the basis for regulating S6K1 peptides productions) of regulating ribosomal activities while the second substrate needed for S6K1 production is the PS/T-Guanosine Kinases.

The availabilities of proline (Pro), Arg, Ser, Thr, Leu, and Tyr amino acids in S6K proper peptides and in Thymine kinases with cytosine kinases will enhance the proper activity of S6K1 and consequently 
enhance the proper functions of ATPase which regulate antiinflammatory processes. ATPase synthesis and GTPase can promote OPA1 inner membrane repair which promote OPA1 oxidative processes that will promote anti-inflammatory processes which prevent cancer, coagulation, platelets aggregation, prevent bones weaknesses, and prevent diabetes diseases symptoms. $\mathrm{Na}+, \mathrm{K}+-\mathrm{ATP}$ ase membrane bonding enzymes (MBE) adopt hypertension, where, the N'A+, K+ ATPase (MBE) functions is stabilizing cells membranes signals activities and adopt the negative charges signals transmission that adopt and stabilizing brain activities. Estrogen biosynthesis depending on Ser/Thr FOX phosphorylation signaling pathways for glucocorticoids synthesis (estrogen is the substrate of GCs synthesis) Upon OPA1 enzymes effects on Estrogen, but the feedback of GCs to produce estrogen upon ATPase and synthetase regulations on GCs isoforms. S6K1 is the regulator kinases for ATPase productions which regulate Interferons (IFNs) isoforms synthesis where IFNs which regulated mainly by pyrimidine-kinases productions from Ser/ Thr phosphorylation signaling pathway. Asthma is characterized by Pre-diabetes and diabetes, that the reasons of causing the two disease are the deficiency in Ser amino acids in the Ser/Thr phosphorylation pathways that reflect deficiency in the pyrimidine kinases (PS/TCytosine Kinase \& PS/T-Thymine Kinases) productions. Where, positively Asthma is characterized by diabetes symptoms but may in the early days of Asthma will not be detected but after early time can be detected. MHC-class-I is (regulated by IFN-gamma) for regulating MHC class-II production which will promote SIRPa synthesis for TLR4 productions, where both SIRPal and TLR4 are regulated by phospholipase effects for regulating endocytic proliferations. Signal regulatory protein-alpha, SIRP-isoforms are contributing for producing IFN-beta then IFN-alpha which activate TLR4 for Plasma membrane synthesis and for proliferations, where through feedback will produce firstly MHC class II (upon synthase betaoxidation) followed by gamma-oxidation for producing MHC Class-I synthesis. SIRP-gamma (IFN-gamma) productions from myeloid is the first SIRP molecular structure that produced and promote MHC-I synthesis regulated by IFN-gamma for endocytic soluble MHC class II synthesis then for SIRPal and for TLR4 biosynthesis. The role of functions of the OPA1 oxidations cycles is promoting the proper S6K1 production for ATPase for producing and for re-functioning the fattyacyl-CoAs for producing NADH and then ATPase. Where, decreasing or deficiency in S6K1 production will reflect the decreasing in the ATPase and reflect decreasing or mutations in mitochondrial OPA1-oxidations then decreasing in NAD and FAD, that reflect the beginning of many diseases including asthma, diabetes, cancers, etc

\section{Methods and Results}

TCA cycles improves antibody, cytokines and acetyl CoA production [1]. Mitoconderial TCA cycles are a regulated metabolite, which considered as a part from FOX genes activities and depending on Ser/Thr-FOX phosphorylation pathways functions, and on OPA1 enzymes effects on carbohydrate, on lipid, and on lipo-proteins nutrient molecules, where, as Ser/Thr-FOX activity produce the four GPS of kinases (PS/T-Cytosine kinases, PS/T-Thymine Kinases, PS/TGuanosine kinases and PS/T-Adenosine kinases) as mitochondrial enzymes will start their oxidative effect for modifying those four kinases GPS as will producing only three fatty acyl-CoA isoforms (gamma, beta and alpha). The full releasing of PS/TGk and PS/TA kinases GPS depending on Ser/Thr FOX phosphorylation pathways and firstly on ATPase with COX enzymes, that their biosynthesis pathways related and depending on OPA1 enzymes functions and on the necessary TCA cycles for producing purines kinases and acylCoAs full of purines, where, TCA cycle functions involved in the release of PS/T-T-Kinase and PSTC-kinase (mTORC1).

The synthesis of fatty-acyl-CoA-synthetase subunits are depending on synthetase enzymes effects on lipo-polysaccharides (LPS) and on long fatty acids chains for pyrimidines synthesis (cytosine and Thymines) which regulate hydrophobic amino acids [2]. The Ser/Thr FOX phosphorylation pathways functions and ATPase with COX oxidative processes are necessary for producing the necessary four kind of kinases GPS which then followed by mitochondrial enzymes oxidative effects for producing three fatty-acyl-CoA- isoforms started by OPA 1 synthetase enzymes effects (gamma-oxidation) for producing fattyacyl-CoA-synthetase (gamma-subunits) followed by beta-subunits synthesis upon synthase function then followed by phospholipase effects for producing alpha-subunits, that their proper activities are related to and depending on the presence of active purines with pyrimidine (which produced from synthetase functions) for hydrophobic amino acids synthesis. Where some of those necessary amino acids are: Alanine amino acids (GCC, GCA, GCG ) (which necessary for alanine-amino transferase) and necessary for migrating molecules and the synthesis of mTORC1 (P-S/T-Cytosine kinases), Arg (AGA , AGG) (which is necessary for ATPase and GTPase synthesis and reactivities), Ser (AGT, AGC) (which is necessary for both cytosine kinases and thymine kinases synthesis), Thr, leu, (which is so necessary for sestrin synthesis and brain activities) and Tyr amino acids synthesis (which considered as imp hydrophobic amino acids and imp for regulating most of active subunits and genes in the cellular metabolism ), where the purifications and synthesis of purines nucleotides (guanine and adenosine) from TCA cycle is a part of FOX pathways which promote the production of the two kinases adenosinekinases and guanosine-kinases for ribosomal activities repairs and for the mitochondrial OPA1 functions which begin with synthetase effects (gamma-oxidations) for necessary pyrimidine synthesis that promote the hydrophobic amino acids synthesis.

Synthetase enzymes can adopt and regulate TCA cycles through functioning the produced purines for purines kinases synthesis (PS/ TGk and PS/TA kinases) and for hydrophobic acids synthesis which build necessary protein kinases subunits (eg S6K1, and other cytokines productions upon firstly the effects of synthetase enzymes) and can rebuild the fatty-acyl-CoA isoforms subunits synthesis (upon OPA1 oxidative effects). TCA cycle metabolites in particular are centrally important determinants of macrophage metabolic re-programming [3]. It has been reported that Metabolism in immune cells is no longer thought of as merely a process for adenosine triphosphate (ATP) production [4], but I reported that ATPase productions are so necessary for all cells biosynthesis and metabolism specifically immune cells for activating their ATPase productions which necessary for creating their necessary active signals transmission, for stimulating cellular biosynthesis and for GTPase productions which necessary for mitochondrial OPA1 inner membrane repairs.

ATPase productions are so imp started by the formation of S6K1 peptides which is regulated by $\mathrm{P}-\mathrm{S} / \mathrm{T}$-Adenosine-kinases and by P-S/TGuanosine-kinases subunits, and by mTORC1 productions that can be stored in ribosome as $\mathrm{S} 6 \mathrm{~K} 1$ then upon phosphorylation will produce ATPase and GTPase that will be directed for repairing OPA1 inner membrane enzymes which will re-activate proper gamma-oxidation (upon synthetase effects) for producing proper necessary gammasubunits or fatty-acyl-CoA-gamma which regulate Beta-subunits productions upon the synthase enzyme effects (beta-oxidations).

Where, the death of living cells started by broken ribosomes or by analyzing RNAs without repairs which can be done through reproducing ATPase or GTPase from their main stored S6K1, where broken ribosome may can lead mutations or stop enhancing OPA1 for producing acyl-CoA isoforms (due to broken mitochondrial membrane) that can reflect Brocken OPAl oxidative pathways including TCA cycles.

Decreasing in FOX activities or broken ATPase will stop releasing PS/ TGk and PS/TAk kinases (which produced upon ATPase and COX enzyme oxidative processes) that will lead to cells death or pathogenic symptoms. 
Also, notice that Tubulin acetylation which regulated by ATPase which plays important role in cellular activities including improving cell polarities, where tubulin acetylation depending on ATPase with GTPase productions that reflect healthy status of living cells and reflect the production of S6K1 by tubulin acetylation.

S6K1 synthesis and functions can controls tubulin acetylation (due to S6K1 is main regulator active peptides for ribosomal repairs and ATPase productions), hence, S6K1 active subunits can contribute to the Autophagic flux induced by different stress conditions and in different cells. During tubulin acetylation, S6K1 contributes to the flux of autophagy induced by different stress conditions and in different cells, where, this effect appears to be independent of the kinase activity of S6K1 [5]. S6K1 biosynthesis can reflect creations of signals effective transmission which can stimulate and promote gamma-oxidation upon the effects of OPA1 synthetase for producing amino-acyl-CoAsynthstase (gamma-subunits) with enough energy that simplified in the created active signals which can be used for migrating the produced subunits and stimulating the beta-oxidations (upon synthase effects on gamma-subunits).

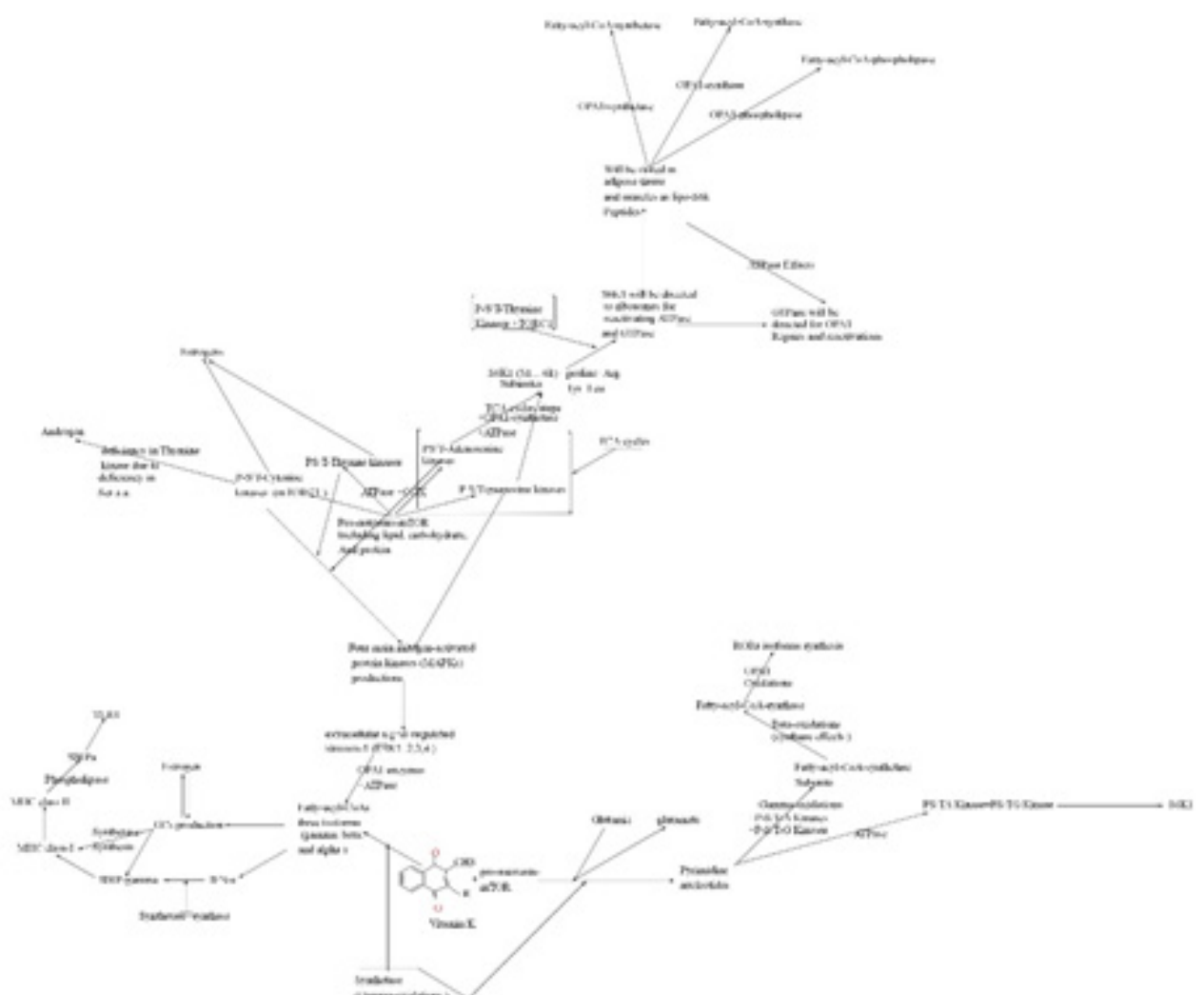

Figure 1: S6K1 synthesis regulated by mTORC1+P-S/T-Thymine kinases productions bonded with purines which produced from TCA cycles.

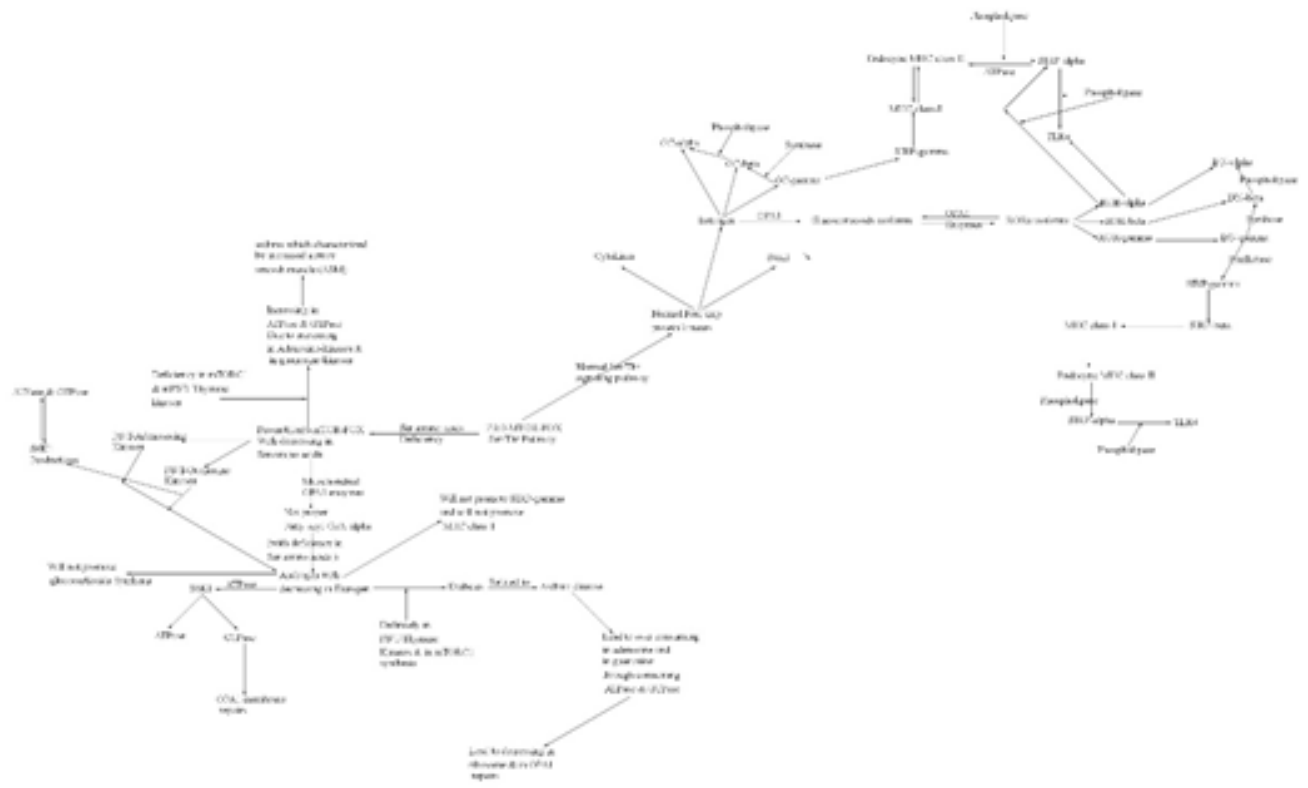

Figure 2: The effects of deficiency of Ser-amino acids in asthma disease \& lead to other diseases symptoms.

GC: Glucocorticoids

IFN: Interferons 
The effects of OPA1-synthetase (OPA1Stase) will regulate pyrimidine synthesis functions (Cytosine and Thymine) and active signals transmission which used for mTORC1 (PSTCk) and other protein kinases subunits productions, where PS/T-Adenosine kinases and PS/ T-Guanosine kinases are necessary for regulating the biosynthesis of the S6K1 peptides, that the produced S6ks from Ser/Thr phosphorylation signaling pathway will be directed for endocytic biosynthesis for repairing and re-activating rRNAs and for ATPase and GTPase productions (where, GTPase is so imp for repairing mitochondrial inner membrane OPA1 ) that OPA1 is necessary for the productions of acyl-CoA isoforms upon OPA1 oxidative processes, and are necessary for controls tubulin acetylation for regulating axon growth through beta-oxidations (for beta-subunits productions) followed by alphaoxidations which regulate alpha subunits productions necessary for axon growth and autophagy biosynthesis.

Also, the conversion of ketoglutarate to glutamate is imp for the acetylated tubulin-NKA complex, and imp for proline synthesis which regulated by TCA cycles.

Effects of ATPase on pro-nutrients-mTOR is for producing protein kinases and S6K1 which can be considered as a basis of variety of cytokines with different compositions.

The mammalian target of rapamycin (mTOR) positively regulates axon growth [6], where, mTORC1 is the main of regulating and stimulating ribosomal S6-protein-kinase-1 (S6K1) synthesis through Fox Serine/ Threonine signalling activated pathways.

And The maintaining of glucose supply for purines synthesis necessary for S6K1 protein synthesis is necessary for central nervous system through for the formation of the three acyl-CoAs (isoforms) for fatty acid synthesis [7]. The maintaining of glucose supply (purines supply) is necessary for reactivating the long fatty acids chains productions upon ATPase effects and COX effects, and for S6K1 synthesis (as I mentioned previously) which necessary for controlling platelet activation and aggregate formation [8].

Maintaining glucose supply (purines supply) are necessary for ATPase reactivation through regulating $\mathrm{S} 6 \mathrm{~K}$ re-production (with proper composition from hydrophobic amino acids example of proline, ser, Arg, Leu and tyrosine) which is necessary for reactivating GTPase synthesis.

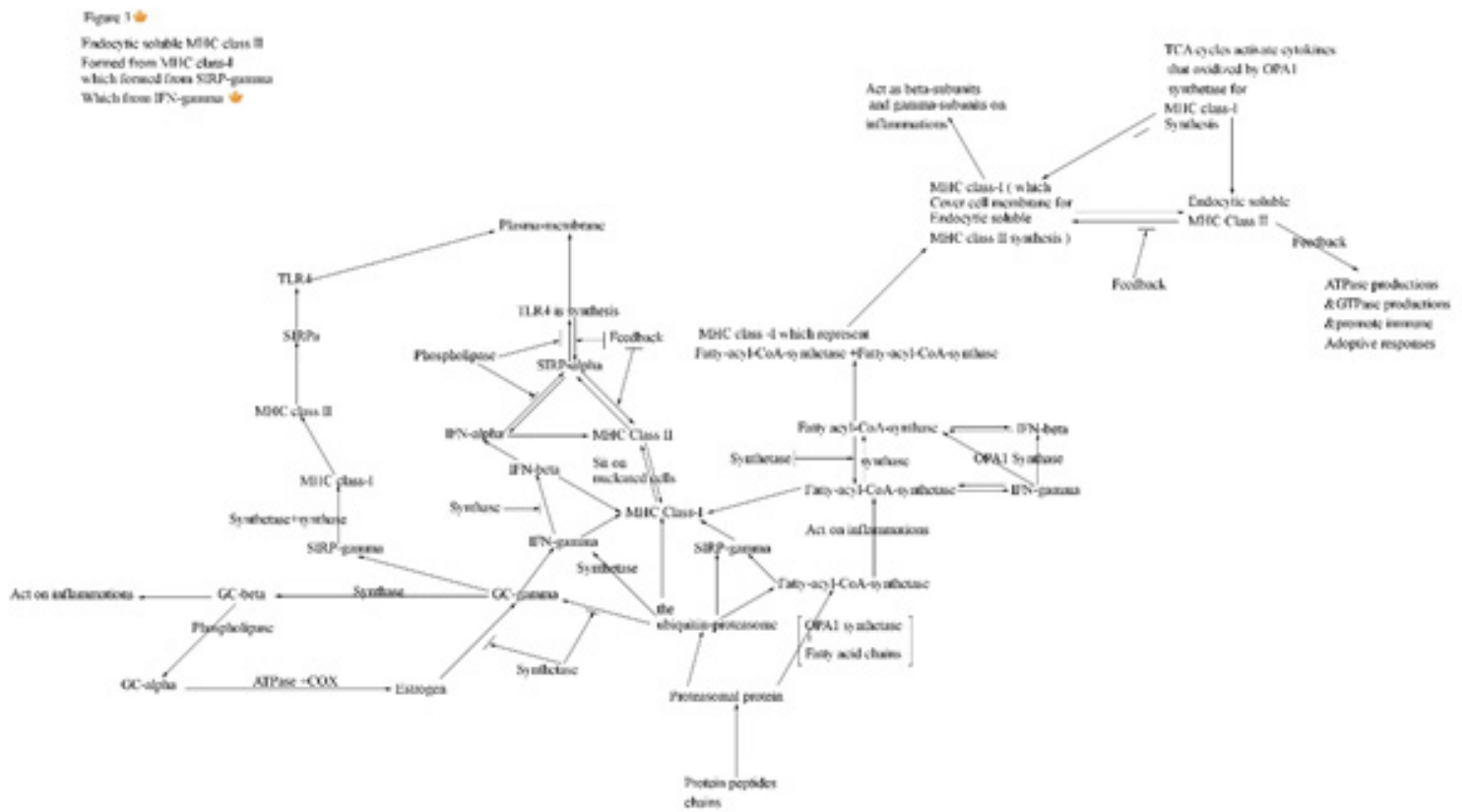

Figure 3: Endocytic soluble MHC class II formed from MHC class-I which formed from SIRP-gamma which from IFN-gamma.

The availabilities of proline (Pro), Arg, Gly, Asp (GAC), Ser, Thr, Leu, and Tyr amino acids are so necessary for S6K proper activities where enhance the proper functions of ATPase and OPA1 oxidative processes (Gamma, beta, then Alpha) which can analyze tumors (upon synthetase effects), coagulation, prevent platelets aggregation, prevent bones weaknesses and erosion of the vertebrae, and prevent diseases symptoms. Also, Asp (GAC) is necessary for deliver its purines $A \& G$ by cytosine to endocytic ribosomes (where, Cytosine imp for tRNAs productions and functions).

The proper ATPase composition is necessary for amphetamine-based anorectic which necessary to maintain neuronal excitability which can rapidly converted into amphetamine in vivo. $\mathrm{Na}+, \mathrm{K}+$-ATPase which is a membrane-bound (MBE) enzyme that can maintain signals activities and maintain $\mathrm{Na}+\mathrm{K}+-\mathrm{ATPase}$ for brain activities [9].

Where, $\mathrm{Na}+, \mathrm{K}+$-ATPase (MBE)is adopting hypertension, and N, A, K, ATPase functions is stabilizing cells membranes signals transmission activities, and adopt the negative charges signals transmission which adopt stabilizing brain activities.

Stimulation of $\mathrm{Na}+\mathrm{K}+-$ ATPase activity (which basically depending on the S6K1 biosynthesis) increases sodium transport across the renal proximal tubule epithelia, [10].

What is the relation of S6K1 to high blood glucose (hyperglycemia) or low blood glucose (hypoglycemia) and diabetic coma? Diabetic disease include either hyperglycemia or hypoglycemia but we should not describe diabetes according to hyperglycemic or hypoglycemic but those phenomena reflect the Consumption, cracking and loss of ATPase in biological processes without normal repairs or with decreasing repairs that can reflect deficiency in pyrimidine nucleotides in S6K1 peptides which is the nain of ATPase synthesis, where the deficiency in Ser amino acids during FOX activities will lead to decreasing in the PS/T-Thymine Kinases and in PS/T Cytosine kinases that will lead to Androgen production instead of Estrogen productions that will reflect mutation in S6K1 peptides due to deficiency in pyrimidines and necessary hydrophobic amino acids in their chains, where S6K1 will be directed to inner cells for producing ATPase (mutated ATPase).

As mutated ATPase started for performing its activities will reflect increasing in Androgen with decreasing in Estrogen, that will lead to increasing in blood glucose (due to increasing in TCA with decreasing in pyrimidine synthesis) which described as hyperglycemia 
But hypoglycemia is the phenomena that describe increasing in mitochondrial oxidative processes with decreasing in proper ATPase biosynthesis, that reflect there are enough early ATPase for performing the catalyzing of sugar molecules but without ATPase repairs that later will be turned to hyperglycemia.

Diabetes is the result of a deficiency in Ser amino acids in the Ser/ Thr signaling pathway that reflect deficiency in the formation of the kinase PS/TTK and PS/TCK (mTORC1), and reflect a decreasing in the formation of pyrimidine nucleotides (which included in Ser amino acids), which leads to the decreasing in the production of Estrogen eith increasing in Androgen synthesis where androgen described as rich of purines (Adenosine and Guanosine) eith decreasing in Thymine and Cytosine nucleotides.

So S6K biosynthesis with deficiency in Ser amino acids will produce a mutated S6K1 peptides with rich of purines and deficiency of pyrimidines that will produce mutated ATPase without normal repair Antibodies are immunoglobulins proteins which can be classified into ribosomal and non-ribosomal peptides and differentiate according to their amino acids Containments (quality and quantity).

Each of those antibody peptides when oxidized by OPA1 enzymes will generate the three fatty-acyl-CoA isoforms (gamma, beta and alpha subunits), that alpha isoforms contain alpha+beta + gamma, but beta isoforms contain beta and gamma isoforms, while gamma contain only gamma isoforms, where each of those acyl-CoA isoforms are necessary for regulating and activating macrophages and T-cells and consequently necessary for myeloid and lymphoid cells activities for proper SIRP productions, for MHC Class-I synthesis which promote the endocytic soluble MHC Class II synthesis. Those necessary acylCoA three isoforms can be functioned directly by OPA1 enzymes for glucocorticoids and for the three interferons isoforms re-synthesis, then for SIRP-gamma production which will be for MHC class-1 which regulate endocytic soluble MHC class II synthesis which will promote the endocytic SIRPa1 synthesis for TLR4 which is necessary for endocytic proliferation roots and activities.

Insulin has two chains $\mathrm{A} \& \mathrm{~B}$ that $\mathrm{A}$ and $\mathrm{B}$ chains become linked together by two sulfur-sulfur (disulfide) bonds. Pro-insulin, insulin, and $\mathrm{C}$ peptide are stored in granules in the beta cells to be released in capillaries [10],

The two A\&B chains are considered as two kinases protein one is " $\mathrm{A}$ " formed from Ser and the other "B" is formed from Thr amino acids. Where, " $\mathrm{B}$ " chain is poor of pyrimidines Containment but rich of purines (that has the ability to recover S6K1 for recovering ATPase and GTPase), while the other chain " $\mathrm{A}$ " is rich of pyrimidines (Thymine and Cytosine) where, both subunits chains are bonded with sulfur=sulfur bonds within beta-cells for beta-cells functions and secretion.

Immune consists of two types of lymphocytes: $\mathrm{T}$ and $\mathrm{B}$ cells, that $\mathrm{T}$ cells can produce a large amount of cytokines through feedback upon the activity of MHC class-I which include and involve synthetase activities, where $B$ cells require specific differentiations and activation conditions to produce cytokines [11]. The beta cells can only produce only kinds of cytokines: 1) PS/T-Thymine Kinase and PS/T-Cytosine kinase from "A" chain, while B chain produce 2) PS/T-Adenosine kinases and PS/T-Guanosine kinases which can

Switching the production of antibody isotypes in B cells, differentiation of helper T cells which regulated by cytokines [12]. B chain has the function of recover S6Ks peptides production depending on the availabilities of amino acids composition.

Antibodies peptides can be classified to three types of antibodies depending on their Compositions from amino acids and fatty acids:

a Type AB-gamma

\section{b Type AB-beta}

c Type AB-alpha

First $\mathrm{AB}$ gamma which include synthetase enzyme that can analyze and destroy beta cells (in the absence of "A" chain) and hepatic cells, but AB-beta activate Beta-cells and IFN-beta and can protect hepatic and beta-cells and can increase anti-inflammations processes (through beta-oxidations),

but $\mathrm{AB}$-alpha can be activated upon phospholipase effects on $\mathrm{AB}$ beta for accelerating proliferation through producing the endocytic SIRP-alpha for promoting the endocytic TLR4 synthesis that regulate plasma membrane synthesis and endocytic proliferations processes.

When, pyrimidines decreased in nutrition in vivo, the $\mathrm{AB}$-alpha will be enhanced by both synthase and synthetase specifically by synthetase for reactivating gamma-oxidations for reactivating pyrimidine synthesis for hydrophobic amino acids synthesis for promoting and recover the "A" chain synthesis

Which can regulate " $B$ " chains in insulin in beta-cells that regulated by synthase effects for Beta-subunit synthesis for AB-beta synthesis which can activate B-cells for promote the GC-beta productions, and for IFN-beta productions.

Then IFN-beta will regulate MHC-class-I productiins and then the endocytic MHC class II synthesis which will promote the endocytic SIRP $\alpha 1$ synthesis upon the phospholipase effects, SIRP $\alpha 1$ will promote the TLR4 synthesis respectively.

That the alpha-oxidations is the necessary processes needed for proliferation including SIRPal productions which necessary for TLR4 synthesis and regulations.

B lymphocytes (B-cells) can activate a type of white blood cell through the of stimulations of beta-oxidations by OPA1 synthase enzymes (that its molecular compositions rich of pyrimidines in hydrophobic acids). It has been reported that preventing hepatic insulin resistance via the adenosine monophosphate-activated protein kinase-p70 ribosomal S6 kinase-1 pathway. [13]

The preventing insuline resistance will need to re-activate protein p70Kinases for renew the S6K1 molecular composition which will recover the beta-chain in insuline for preventing insulin resistance. S6K1 is necessary for insulin growth that as I mentioned before two types of protein kinases responsible for S6 productivity through Ser /Thrneutrient-mTOR-FOX signaling pathway which are PS/T-Adenosine kinase and PS/T-Guanosin kinases which are mainly produced from Thr amino acids phosphorylation which are rich of purines for S6K1 synthesis then for both ATPase and GTPase.

Hence, S6K1 can activate insulin growth and vice versa. That, the insulin-induced activation of p70 S6 kinase and MAP kinases [14]. Where, amino acids are necessary for the insulin-induced activation of $\mathrm{mTOR} / \mathrm{S} 6 \mathrm{~K} 1$ signaling and protein synthesis to prevent insulin resistant in human skeletal muscle [15].

The insulin resistance or drugs resistance occur due to stability of specific fragments due to increasing in positive linkages that stabilize linkages against antibiotic can resist OPA1 oxidative processes which have to be occurred for renew those +ve fragments for continuing adopting the necessary molecular structure for proper endocytic processes. The resistance to antibiotics can be due to deficiency in Ser amino acids and in pyrimidine nucleotides with decreasing oxidative processes by mitochondrial enzymes, but some cases can be due to reductions in ATPase with reduction in $\mathrm{S} 6 \mathrm{~K} 1$ productions due to decreasing in ser/Thr signaling pathway.

The Containments of specific advantages amino acids as Proline in gamma, beta, and alpha subunits can accelerate proper ATPase 
activities and OPA1 oxidations which facilitate ifns isoforms productions for MHC-class-I synthesis which regulate the endocytic MHC class II synthesis for SIRPal production which necessary for TLR4 synthesis and TGF-gamma/beta/\& alpha synthesis respectively. Where the presence of proline in IFN-gamma, in GC-gamma, in TLR4 genes and in IFN-beta will accelerate their activities and oxidative OPA1 anabolic processes and accelerate the alpha oxidations for SIRPa1 and TLR4 production productions which promote plasmamembranes, collagen synthesis and blood platelets, [16] CD8 T cells as well as other cells can and do produce IFN- $\gamma$ during M. tuberculosis infection. [17]. S6K1 consider as a mediator subunits for Interferons (IFNs) isoforms synthesis regulated by pyrimidine-kinases. It has been reported that interferons are a family of cytokine mediators critically involved in alerting the cellular immune system to viral infection of host cells [18].

Interferons are not alerting central immune to viral infections, but interferons are produced for modulating the anti-inflammatory processes (not alerting original basic genes and subunits) which in brief through modulating IFNs synthesis and glucocorticoids biosynthesis, and for Interferons isoforms synthesis.

Ribosomal peptides S6 kinase beta-1 (S6K1) is a result from Serine/ Threonine phosphorylation pathway, that has imp roles in immune regulating or promoting the lymphocyte activations, where S6K1/S6 axis participated in the primary response of anti-bacterial adaptive immunity in Nile tilapia [19].

And, Interferons-Dependent Engagement of Eukaryotic Initiation Factor 4B via S6 Kinase (S6K)- and Ribosomal Protein S6KMediated Signals [20]. The mRNAs translation for IFN-sensitive clearly are the S6K1 peptides that activate ATPase phosphorylation which back phosphorylate the eIF4B on Ser amino acids for adopting IFN gamma, beta, and alpha compositions through translations according to the basic S6K1 and then related to ATPase sequences composition.

Any mutations will occur in S6K1 peptides due to absence of Ser, Arg, proline, Thr, tyr or leu or due to absence or decreasing in pyrimidines can reflect pathogenic symptoms. Asthma is characterized by Prediabetes and diabetes, that deficiency in pyrimidines (deficiency in Ser) is the main reason for two disease.

It has been reported that (S6K1) inhibition with rapamycin inhibited IFN- and EGF-induced protein synthesis [21]. In severe Asthma which characterized by increased airway smooth muscle (ASM) that begin due to decreasing in Ser amino acids in pro-nutrients-mTOR-FOX pathways that will reflect decreasing in the PS/T-Cytosine-Kinases (mTORC1) and in PS/T-Thymine-Kinases production (which are necessary for migrating and regulating molecules too) with increasing in $\mathrm{P}$ Ser/Thr-Adenosine-kinases with Ser/Thr-Guanosine-kinases (which specified for S6K1 peptides synthesis) that lead to decreasing in migrating molecules due to decreasing in cytosine (decreasing in pyrimidines), and decreasing in tRNAs with increasing in ATPase and GTPase (which repaired and reactivated by S6K1 peptides) that will lead to promote glucocorticoids (from androgen) and interferons synthesis, where their biosynthesis characterized by deficiency in Ser amino acids and in its related kinases (PS/T-Cytosine Kinases and PS/T-Thymne Kinases) productions, that will lead to decreasing in sugar, lipid, LPS, and carbohydrate metabolism (lead to increasing of purines molecules and LPS in blood and interstitium fluid) and conjugated with increasing in S6K1 peptides productions (for ATPase repair) and for growth factor synthesis, that will lead to increasing in airway smooth muscle (ASM), note, patients that having Asthma are having the so high risk of sever diabetic problems due to deficiency of Ser amino acids (deficiency in Ser/Thr phosphorylation pathways) that will lead to deficiency in Estrogen productions with increasing in Androgen productions which are characterized by high purines with deficiency in pyrimidines nucleotides (deficiency in pyrimidinekinases lead to deficiency in PS/T-Cytosine Kinase and PS/T-Thymine kinases synthesis).

Consequently deficiency in pyrimidine can lead to mutations in ribosimal enzymes and mutations in the productions of long fattyacyl-CoA-isoformes upon OPA1 enzymes functions. So, Asthma are characterized by diabetes symptoms, where, in the early days of Asthma diabetic symptoms cannot be detected but later will be detected.

Where, there are limited data on the risk of pulmonary disease in patients with diabetes [22]. And, it is concluded that asthma and T2DM are the two common chronic conditions of increasing prevalence and that often coexist in the same patient [23].

Also, it has been reported that: Prediabetes and diabetes have been recently identified as risk factors for asthma exacerbations in adults [24]. Note, in some cases decreasing in Cytosine-kinases and or fattyacyl-CoA-synthetase (gamma-subunits) will reflect decreasing in ATPase repairs (due to cytosine necessary with purins for rebuilding ATPase which is necessary for migrating molecules) that lead to accumulations of subunits within cells and in the interstitium fluid that lead to decreasing in their migrations with increasing in their +ve linkages that lead to tumor growth and coagulation in blood vessels and can reflect other diseases symptoms example: early diabetic symptoms. Where, Advances along the small GTPase front have implicated cell migration. [25]

Proper GTPase productions implicate cell migrations and the mitochondrial OPA 1 membranes repairs in case of availabilities of both PS/T-Cytosine kinases and PS/T-Thymine Kinases (and in availability of proline and hydrophobic amino acids that accelerate OPA1 anabolic oxidative processes properly) that can increase Tyrosine metabolic pathways and will increase signals transmissions that will promote the migrations of active of genes and subunits which can activate betaoxidative processes and alpha-oxidations (that regulated by synthase and by phospholipase respectively), that can promote phosphatidic acid (PA) to rebinds to mTOR and to re-binds to S6K peptides independently of mTOR, where S6K peptides synthesis are basically depending on the two purines kinases that produced from Ser/ThrFOX signaling pathways for producing (PS/T-Adenosine $\mathrm{k}$ and PS/ TGuanosine kinases for S6K1 synthesis, for ATPase and for GTPase reactivities, and for promoting endocytosis MHC class II functions, where its migration need the availabilities of Cytosine-kinases and Thymine-kinases (active pyrimidine-kinases) production for S6K1 peptides productions and for tRNAs synthesis. MHC Class-I is fattyacyl-CoA gamma-subunits (IFN- gamma) for modifying their fatty acyl-CoA-beta (IFN-beta) synthesis to be site and lie on nucleated cells as MHC class-1.

The IFN-gamma biosynthesis regulated by the effects of synthetase enzymes on lipid and LPS for producing long-fatty acids chains (through gamma-oxidation) for producing fatty-acylCoA-synthetase subunits (IFN-gamma) that promote IFN-beta, where both IFNgamma and beta have the functions of activating the soluble endocytic MHC class II synthesis which originally produced from MHC class-I and has anti-inflammatory activities that will migrate to nucleated cells to site and lie on their membrane for producing endocytic MHC class II.

The biosynthesis of long fatty-acyl-CoA-synthetase (ACS) and other beta and alpha isoforms are acting as homodimeric enzymes, that acyl-CoA synthetase member 6, ACSL6, is a form present in the plasma membrane of cells. [26]

Where, "ACS" productions are necessary for regulating TLR4 which necessary for plasma membrane synthesis, and necessary for 
endocytic MHC class II synthesis which originally regulated by fattyacylCoA-gamma (synthetase functions) and by IFN-gamma that carry the anti-inflammatory function that bind to inflammation contents for modifying their own gamma-subunits for for regulating MHC-class-I which followed by the endocytic MHC class II synthesis that is so necessary for endocytic SIRPa1 productions.

IFN-gamma has the functions of anti-inflammatory processes that can analyze inflammation and infected cells for binding to resulted peptides and recreate pyrimidine nucleotides for modifying its own active gamma-subunits and then for regulating IFN-beta synthesis upon the effects of OPA1-synthase enzyme followed by the regulation of MHC-class-I which regulate the endocytic MHC class II synthesis followed by SIRPa1 and TLR4 synthesis respectively.

That MHC class II can be described as the main regulator for reactivating the endocytic TLR4 upon phospholipase. Furthermore, the co-expression of MHC class II molecules and TLR2 or TLR4 in human embryonic kidney (HEK) cells 293 leads to enhanced production of the anti-microbial peptide human- $\beta$-defensin [27]. Previous study indicating the imp fact that MHC class II enhance the endocytic TLR4 reactivities upon phospholipase oxidative effects. Where, MHC-II molecules are important for initiation the antigenspecific immune response where need to be promoted by activating TLR4 for endocytic proliferation then for external proliferations.

Dendritic cells (DCs) initiate adaptive immune responses by activating $\mathrm{T}$ cells via cognate interactions between MHC-peptide complexes and $\mathrm{T}$ cell receptors. [28] where, class II is predominantly stored in endocytic plasma membrane, where it has a short half-life because of its regulation to TLR4 synthesis for Plasma membrane modification and re-synthesis, that is why the plasma membrane which promoted by MHC Class II and formed by TLR4 its Containment is MHC class II, and It has been considered that $\mathrm{T}$ cell growth-promoting activity of interferon-gamma [29]. And Also, IFN $\gamma$ mediates CD8 T-cell cytotoxic function. That in vivo, antigen-specific CD8 T cells that produce INF $\gamma$ necessary to effect rejection of skin grafts [30], where, cytokine productions upon FOX activities will be modified by the effects of mitochondrial enzymes to produce specific modified IFN-gamma which has the anti-inflammatory effect and binding to selected of result peptides that will reactivate IFN-beta upon synthase oxidations and then will site on and cover nucleoid cells for MHC class II synthesis which will has the role of reactivating TLR4 upon regulation of phospholipase for Plasma membrane synthesis and for TLR4 other functions. Also, The ability of CD8 T cells to re-produce IFN $\gamma$ indicating that IFN $\gamma$ connected to the MHC class II re-activities, and in fact as mentioned above that IFN- $\gamma$ basically has the function of regulating the MHC class II synthesis that during inflammation and infection the IFN $\gamma$ will analyze inflammations content through gamma-oxidation then will bind to selected resulted peptides for modifying its own subunits then will sit and lie on cell membrane as MHC class-I for reactivating the endocytic soluble MHC class II synthesis, also would like to mention that MHC class II is a fattyacyl-CoA-synthase (Beta-subunit) which regulate and activate TLR4 synthesis upon phospholipase oxidations for producing fatty-acylCoA-phospholipase (alpha-subunits which considered as IFN-alpha necessary for proliferation cycles), that the interferons (IFNs) have functions and diverse effects upon OPA1 oxidations on initiate and adapting immune cells during infection including proliferations function through regulating TLR4 for running proliferations.

In conclusion the previous studies indicated that MHC class-I considered as fatty-acyl-CoA-synthetase (gamma-subunits) and considered as IFN-gamma that can analyze infected cells and inflammations then bind to their peptides for modify and promote IFN-beta synthesis upon synthase oxidations covering nucleated cells membranes for reproducing MHC class II which cobsidered as fatty-acyl-CoA-beta (IFN-beta) that upon alpha-oxidations will promote fatty-acyl-CoA-phospholipase which considered IFNalpha for regulating TLR4 synthesis upon alpha-oxidations for plasma membrane synthesis which contain MHC class II , and then again act on inflammations and on infected cells through feedback for reproducing IFN-beta then IFN-gamma regulated by OPA1 oxidations across cells membrane for acting on inflammation and bind again to their peptides for re-improving their gamma-subunits and Betasubunits (which conclude gamma-subunits in their chains) which can re-modified again and act as MHC class-I which will re-sit and lie on cells membranes for running the soluble endocytic MHC class II synthesis and then TLR4 synthesis.

Where, Thr, Gly, Glu, Arg, Lysine (AAG, AAA), and proline are the most important amino acids peptides for rebuilding necessary fattyacyl-CoAs isoforms (IFN-isoforms) and for necessary MHC class II that can be stored in lysosomes, for running OPA1 oxidative processes and for TLR4 synthesis to perform proliferations, for adopting neurons response, and for promoting brain cells functions, where MHC class II is regulated by MHC class-I synthesis.

On the other hand, the Tyr, Met, Arg, Ser, Pro, and Leu amino acids are so necessary amino acids for rebuilding the proper MHC class- 1 which regulate and promote MHC class II synthesis and then regulate both SIRP $\alpha 1$ and TLR4 biosynthesis for endocytic plasma membrane synthesis and endocytic proliferations.

Signal regulatory protein $\alpha$, (SIRPa) is necessary for IFN-alpha synthesis which activate TLR4 for Plasma membrane synthesis where through feedback will produce firstly MHC class II (upon betaoxidation) followed by gamma-oxidation for producing MHC Class-I.

Signal regulatory protein-alpha (SIRPa1), also known as Src homology 2 domain-containing phosphatase substrate- 1 , is expressed on myeloid and hematopoietic stem cells and neurons. Where the production of SIRP $\alpha$ and their activities can increase signals transmissions that can increase migrations and signal transduction, and promote OPA 1 alpha oxidations, for cell proliferation, and transformation.

SIRPa has the function of producing IFN-alpha and TLR4 that through feedback will produce firstly endocytic MHC class II and IFN-beta (SIRP-Beta) upon synthase beta-oxidation, then followed by MHC Class-I and IFN-gamma productions (also considered as SIRPgamma) upon synthetase oxidations that will sit and lie on nucleated cells membranes recreating the MHC class II again and for running the anti-inflammatory functions and internal endocytic activities.

Signal regulatory protein a negatively regulates mast-cell activation [31]. And Ligation of SIRPa (on macrophages) by CD47 (on RBCs) promotes tyrosine phosphorylation [32].

SIRPa is negatively regulate mast cells directly but can activate mast cells indirectly through SIRP-ganma production that can reactivate both IFN-gamma and MHC-class-I synthesis (by stem cells), furthermore the SIRP that can promote Tyrosine phosphorylation due to the gamma-oxidation upon synthetase enzyme that activate SIRP-gamma from both beta and alpha subunits that can promote Tyrosine phosphorylation pathways which promote pyrimidine and hydrophobic acids synthesis (Tyr, Leu... etc).

The IFN- $\gamma$-induced surface expression of CD47 contributed to a stronger binding affinity to SIRPa. [33]. Where, IFN- $\gamma$ have the function of promoting MHC-class-I which directed to the nucleated cell membrane for promoting endocytic MHC class II, while has the function of promoting the SIRP-gamma which promote the MHCclass-I too.

And this study indicate that The two subsets of CD14+SIRPa had limited ability to migrate and phagocytose; but stimulated T-cell 
function [34], where, SIRPa has the activities of promoting TLR4 synthesis and proliferation but not migration, while, SIRP-gamma has the activation for increasing signals transmission and migrations.

2'-5'oligoadenylate synthases (OASs), a family of latent 2'-5'-adenylyl transferases, otherwise involved in cellular antiviral responses, are also involved in PAR remodeling of the DDR in MDS and AML cells [35]. Myeloid cells are major players that exploit the regulators of Arginine metabolism (for proline synthesis) to mediate diverse, and adopt immunity [36]. That fatty-acylCoA-Synthetase in myeloid is so important for adopting TCA cycle (Arg metabolism) which has the function of purines synthesis and proline synthesis, while also synthetase regulate pyrimidine nucleotides synthesis for acyl-CoA synthetase productions and for IFN-gamma productions.

In conclusion I would like to declare imp fact that SIRPa is produced from the endocytic MHC class II and from endocytic IFN-beta which can promote TLR4 synthesis, while SIRP-gamma is secreted from IFN-gamma in myeloid and hematopoietic stem cells and neurons that promote MHC-class-I synthesis and promote the endocytic SIRPal production, and has not the ability to activate mast cells that is secreted as so active SIRPGamma sensor "fatty-acyl-CoA-synthetase" that modified by Arg metabolism.

SIRP-gamma upon synthase effects will promote SIRP-2-Beta upon regulations effects of synthase, where both SIRP-gamma and beta will promote the MHC-class-I production which migrated to nucleated cells membranes to sit and lie on for promoting the endocytic soluble MHC class II which upon alpha-oxidations by will produce SIRPa (IFN-alpha) Which will be directly promote TLR4 synthesis for modifying plasma membrane and for necessary endocytic proliferations.

The metabolic purpose of fatty acid oxidation is to generate NADH for ATP generation by the regulations of mitochondrial OPA1 membrane oxidative phosphorylation effect [37]. And, FFAs are activated via esterification to CoA, which generates a fatty acyl-CoA moiety [38] Fatty acid oxidation produce acetyl-CoA units which move in this pathway as fatty acyl-CoA derivatives for utilizing NAD and FAD [39]

So, When OPA1 oxidations is absent it will prevent the fatty-acylCoA synthesis and then will increase fatty acids accumulations due to decreasing in the OPA1 oxidations and will decrease or prevent the production of NAD and FAD and consequently reduce Vitamin $\mathrm{K}$ metabolic cycle and ATP productivity, that decreasing on ATPase will decrease the lipid, carbohydrate, and protein metabolism through decreasing in FOX pathways and then decreasing in ROR-gamma then decreasing in ROR-beta then decreasing in ROR-alpha which lead to decreasing in proliferation and deficiency in immune functions.

$\mathrm{NADH}$ and FADH2 and then the TCA cycle are activated by the electron transport signal transduction which yield from gammaoxidation processes, where NADH and FADH2 produced by both $\beta$-oxidation and the TCA cycle are using the electron transport chain to produce ATP [40].

Where, NAD+ deficiency has been found in models of a number of diseases such as cerebral ischemia, myocardial ischemia, and diabetes, and in models of aging NAD+ deficiency is a common central pathological factor in a number of diseases [41].

The decreasing or deficiency in S6K1 production will reflect the decreasing in the ATPase which also reflect the decreasing in mitochondrial OPA1-oxidations and decreasing in NAD and FAD that reflect diseases symptoms as Asthma, diabetes, cancers, etc, where, NAD+ treatment has been shown to reduce PARP1-induced astrocyte and prevent PARP1-mediated NAD+ depletion in cardiac myocytes in the presence of $\mathrm{H} 2 \mathrm{O} 2$ [42].
NAD is so necessary for MHC class-I synthesis where, The degradation of most cellular proteins occurs by the ubiquitin-proteasome pathways peptides generated by the ubiquitin-proteasome pathway which are presented by MHC class I molecules [43]. So, NAD and proteasomal pathway has their strong effects on most of proteins degradations and their synthesis which can be necessary for rebuilding MHC class-I subunits which promote endocytic MHC class-II productions.

Estrogen formed from Ser/Thr mTOR Fox signaling pathways for glucocorticoids synthesis. Upon OPA1 enzymes effects on estrogen, but the feedback of GC to produce estrogen will upon ATPase \& cox on GCs isoforms: Fatty acyl-CoA synthetase) that can be used for re-activating glucocorticoid-gamma productions, while the effects of synthase on acyl-CoA-synthetase (gamma subunits) will produce glucocorticoid-beta which can produce IL-beta that reactivate astrocyte, netrin-1, and neutrophiles functions. The glucocorticoid Receptor (GRs) has roles of function of re-activating steroid hormone upon ATPase and cox via ser Thr Fox signaling pathways, and mitochondrial matrix repair and activations, throughout reactivate S6K1 synthesis for ribosomal ATPase and GTPase productions which necessary for OPA1 repair, where glucocorticoid (GC) contains the estrogen receptors that GR has strong roles for estrogen biosynthesis and vise-versa in different pathways the first through ATPase Effects via Ser/Thr FOX pathways for re-producing estrogen, but second through OPA1 oxidative processes on estrogen, and strong roles in antiinflammatory pathways.

The glucocorticoids isoforms (GCs) Biosynthesis pathways is linked to Estrogen biosynthesis and protein kinases which promote Estrogen synthesis. The effects of synthetase on estrogen is for analyzing Estrogen molecules for producing glucocorticoids-gamma subunits and utilizing active signals for re-activating glucocorticoids-beta synthesis upon synthase effects on GC-gamma. GC Gamma is the basic units for netrin-1 synthesis which is the basic for the neutrophile repairs and reactivations.

Glucocorticoids synthesis begin from estrogen upon effects of synthetase for GC gamma production, followed by beta-oxidation by the effects of synthase for producing glucocorticoids-beta and others Beta-subunits that can show enhancing and increasing in the uptake of other beta a myeloid protein [44] followed by phospholipase effects in case of proliferations processes through producing $\mathrm{GC}$ alpha.

Glucocorticoid-gamma has anti-inflammatory activities on inflammations for producing pro-inflammatory subunits due to the effects of acyl-CoA synthetase (gamma subunits) on inflammations for analyzing their contents for starting anti-inflammatory pathways activity, and also GC-beta has the same anti-inflammation effect and has the function of SIRP synthesis from myeloid for the MHC class-I production which directed to nucleated cells to lie on and produce the endocytic MHC class II for the SIRPa production and for TLR4 synthesis . GC- beta productions regulate GC-alpha which regulate membrane receptors through activating TLR4 started by SIRPa production. Brocken Estrogen by synthetase can activate a myeloid Beta-subunit synthesis.

In case of diabetic disease is androgen can produce glucocorticoids and vise-versa? In case of androgen synthesis due to deficiency in Ser amino acids lead deficiency in Ser/Thr-Fox signaling pathway will not produce GC gamma or GC beta upon ATPase Effects

(depending on the \% of Ser deficiency) but will produce GC alpha that also can produce SIRPal and TLR4 eith a deficiency in their pyrimidine nucleotides, but will not produce proper MHC class-I and also the MHC class II will not be produced in nucleated cells that diabetic patients will have a deficiency in T-cells re-activations and in macrophages reactivation. Also, Androgen in diabetes disease can increase mainly the S6K1 resynthesis and ATPase productions and 
reactivities due to the Androgen is rich of purines and at the same time can increase not proper SIRPa and TLR4 for only reactivating ribosomes and OPA1 repairs with reincreasing in S6 synthesis. Estrogen avaliablities can reactivate OPA1 synthetase activities that can promote pyrimidines production for hydrphobic amino acids synthesis, while Androgen cannot promote and cannot activities synthetase functions that can't activate hydrophobic acids synthesis, also has not the abilities to produce both proper GC gamma, beta and SIRP, consequently androgen cannot activate MHC class-I productions and Supress IFN gamma productions [45].

\section{Discussion and Conclusion}

S6K1 consider as protected basic subunits for ribosomal ATPase, and for Interferons (IFNs) isoforms synthesis regulated by pyrimidinekinases. The Proper GTPase implicate cell migrations regulated by PS/T-Cytosine kinases and PS/T-thymine Kinases (in availability of proline and hydrophobic amino acids for promoting OPA1 anabolic oxidative processes properly) that can increase Tyrosine metabolic pathways and increase signals transmissions that can promote the migrations of active of genes and subunits which activate betaoxidative processes and alpha-oxidations (that regulated by synthase and by phospholipase respectively), that can promote phosphatidic acid (PA) to re-binds to mTOR and to re-binds to S6K peptides independently of mTOR-FOX pathways .

Basically MHC class-I considered as fatty-acyl-CoA-synthetase (gamma-subunits) that regulated by IFN-gamma that promote and regulate SIRP-gamma productions, and promote IFN-beta synthesis upon synthase oxidations. MHC-class-I can migrate to nucleated cells membranes to promote endocytic MHC class II that upon alpha-oxidations will promote IFN-alpha and SIRPal synthesis for promoting TLR4 synthesis upon the alpha-oxidations for plasma membrane synthesis and for endocytic proliferation.

IFN-gamma regulate both MHC-class-I and SIRP-Gamma sensor "fatty-acyl-CoAsynthetase" that modified by Arg metabolism that stimulate and promote anti-inflammatory processes, and regulate the productions of SIRP-2-Beta (IFN-beta) upon regulations of synthase which activate MHC-class-I production to be directed to nucleated cells membranes to sit and lie on for producing MHC class II that will promte ad SIRP-Beta and SIRPa (IFN-alpha) which promote TLR4 synthesis for modifying plasma membrane and for necessary proliferations when upon feedback will reproduce the MHC class-I.

The decreasing or deficiency in S6K1 productions will reflect the decreasing in the ATPase (can be detracted after consuming the stored adenosine nucleotides in producing ATPase) and reflect decreasing in fatty acyl-CoAs production which regulated by OPA1 oxidative function that can reflect decreasing in NAD and FAD which can start the Asthma, diabetes, cancers diseases.

The effects of synthetase on Estrogen will activate glucocorticoids isoforms synthesis start by GC-gamma productions followed by glucocorticoids-beta upon synthase effects on GC-gamma. GC Gamma is the basic units for netrin-1 synthesis which is the basic for the neutrophile repairs and reactivations.

SIRP-gamma regulated by IFN-gamma where both regulate MHC class-I synthesis which will be migrated to nucleated cells for MHC class II that will regulate SIRP-beta synthesis which upon alphaoxidations by phospholipase effects will produce SIRPa that will be promote TLR4 synthesis. Estrogen is the substrate for glucocorticoids biosynthesis that GCs isoforms can recover IFNs isoforms and viceversa.

Myeloid cells are major players that exploit the regulators of Arginine metabolism (for proline synthesis) to mediate diverse, and adopt immunity. That fatty-acylCoA-Synthetase in myeloid is so important for adopting TCA cycle (Arg metabolism) which has the function of purines synthesis and proline synthesis, and also synthetase control the pyrimidine synthesis for hydrophobic amino acids synthesis Tyr, Leu, Ser, Arg, Pro, etc.

\section{Conflict of Interest}

The Author declare that the research work has been conducted in the absence of any commercial or financial relationships, that could be construed as a potential conflict of interest.

\section{References}

1. Zhang, X, Rubin J, Henry L, Sen X (2020) Feeding tricarboxylic acid cycle intermediates improves lactate consumption and antibody production in Chinese hamster ovary cell cultures. Biotechnol Program 36(4): e2975.

2. Ashraf Marzouk El Tantawi (2021) OPAl is basis for macrophages, glucocorticoids subunits, neutrophile, RAW264.7 Cells and T-Cells regulations, where OPA1 contain COX sequence followed by synthetase, then synthase, then phospholipase sequences respectively. International Journal on Pharmacy and Sciences 1(2): 52-61.

3. Jordan T Noe, Robert A Mitchell (2019) Tricarboxylic acid cycle metabolites in the control of macrophage activation and effector phenotypes. Journal of leukocyte Biology 106(2): 359-367.

4. Niamh C. Williams, Luke AJ O'Neill (2018) A role for the krebs cycle intermediate citrate in metabolic reprogramming in innate immunity and inflammation. Frontiers in Immunology.

5. Aleksandra Hac, Karolina P, Anna HA (2021) S6K1 is Indispensible for stress-induced microtubule acetylation and autophagic flux. Cells 10(4): 929.

6. Hassan Al Ali, Ying Ding Y, Tatiana S, Wei W, Yan S, et al. (2017) The mTOR substrate S6 kinase 1 (S6K1) is a negative regulator of axon regeneration and a potential drug target for central nervous system injury. The Journal of Neuroscience 37(30): 7079-7095.

7. SS Devi (2010) Structure and function of hepatic parenchymal cells Comprehensive Toxicology p. 11-30.

8. Josef E Aslan, Garth WT, Cassandra PL, Jiaqing P, Owen JTM (2011) S6K1 and mTOR regulate Racl-driven platelet activation and aggregation. Blood 118(11): 3129-3136.

9. Gislaine T Rezin, Emilio L Streck, Cinara LG, Gabriela KF, Mariane RC, et al. (2014) Evaluation of Na+, K+-ATPase activity in the brain of young rats after acute administration of fenproporex. Brazilian Journal of Psychitary 36(2): 138-142.

10. Ariel Jaitovich, Alejandro M Bertorello (2010) Salt, Na+,K+-ATPase and hypertension. Life Sciences 86(3-4): 73-78.

11. Monica I Vazquez, Jovani CD, Albert Zlotnik (2015) B cells responses and cytokine production are regulated by their immune microenvironment. Cytokine 74(2): 318-326.

12. Stuart G Tangye, Anthea F, Danielle TA, Cindy S Ma, Philip DH (2002) Isotype switching by human B-cells Is division-associated and regulated by cytokines. The Journal of Immunology 169(8): 4298-306.

13. Eun Ju Bae, Yoon Mee Yang, Jin Wan Kim, Sang Geon Kim (2007) Identification of a novel class of dithiolethiones that prevent hepatic insulin resistance via the adenosine monophosphate-activated protein kinase-p70 ribosomal S6 kinase-1 pathway. Hepatology 46(3): 730-739.

14. J Wijkander, $\mathrm{T} R$ Landstrom, $\mathrm{V}$ Manganiello, $\mathrm{P}$ Belfrage, $\mathrm{E}$ Degerman (1998) Insulin-induced phosphorylation and activation of phosphodiesterase $3 \mathrm{~B}$ in rat adipocytes: Possible role for protein kinase $\mathrm{B}$ but not mitogen-activated protein kinase or p70 S6 kinase. Endocrinology 139(1): 219-227.

15. Micah J Drummond, Jill A Bell, Satoshi Fujita, Hans C Dreyer, Erin L Glynn, et al. (2008) Amino acids are necessary for the insulin-induced activation of mTOR/S6K1 signaling and protein synthesis in healthy and 
insulin resistant human skeletal muscle. Clinical Nutrition 27(3): 447456.

16. Ashraf Marzouk El Tantawi (2021) Toll-like receptor-4, synthesis is regulated by JNK signaling, by three glucocorticoids isoforms and by three interferons isoforms, also its advantages in activities depending on the containment from Arg, Proline and hydrophobic amino acids in its compositions. Open access Journal of Biomedical Science 4(4): 11251133.

17. Yoon, Ji-Won, Jun, Hee-Sook (2005) Autoimmune destruction of pancreatic beta cells. American Journal of Therapeutics 12(6): 580-591.

18. M G Baines, J Hiscott, P Génin, M G Baines (2000) Interferon activation and innate immunity. Reviews in Immunogenetics 2(3): 374-386.

19. Kunming Li, Xiaotong Shen, Hong Qiu, Tianyu Zhao, Kete Ai, et al. (2020) S6K1/S6 axis-regulated lymphocyte activation is important for adaptive immune response of Nile tilapia. Fish and Shellfish Immunology 106: $1120-1130$.

20. Barbara Kroczynska, Surinder Kaur, Efstratios Katsoulidis, Beata Majchrzak-Kita, Antonella Sassano, et al. (2009) Interferon-dependent engagement of eukaryotic initiation factor 4B via S6 kinase (S6K)- and ribosomal protein S6K-mediated signals. Molecular and Cellular Biology 29(10): 2865-2875.

21. Elena A Goncharova, Poay N Lim, Amelia Chisolm, Homer W Fogle III, Jerome H Taylor, et al. (2010) Interferons modulate mitogen-induced protein synthesis in airway smooth muscle. Lung Cellular and Molecular Physiology 299(1): 25-35.

22. Samantha F Ehrlich, Charles P Quesenberry Jr, Stephen K Van Den Eeden, Jun Shan, Assiamira Ferrara (2010) Patients diagnosed with diabetes are at increased risk for asthma, chronic obstructive pulmonary disease, pulmonary fibrosis, and pneumonia but not lung cancer. Diabetes Care 33(1): 55-60.

23. Raimeyre Marques Torres, Marcela Dos Santos Souza, Ana Carla Carvalho Coelho, Luane Marques de Mell, Carolina Souza-Machado (2021) Association between asthma and type 2 diabetes mellitus: Mechanisms and impact on asthma control-A literature review. Canadian respiratory journal 20: 1-13.

24. Tianshi David Wu (2020) Diabetes and glycemic dysfunction in asthma. The Journal of Allergy and Clinical Immunology 8(10): 3416-3147.

25. Julian Gomez Cambronero (2010) New concepts in phospholipase D signaling in inflammation and cancer. The Scientific World Journal 10: 1356-1369.

26. Eric Soupene, Nghi Phuong Dinh, Melvin S, Frans AK (2010) Activity of the acyl-CoA synthetase ACSL6 isoforms: role of the fatty acid Gatedomains. BMC Biochemistry 11: 18.

27. Remo Frei, Johanna Steinle, Thomas Birchler, Susanne Loeliger, Caroline Roduit, et al. (2010) MHC class II molecules enhance toll-like receptor mediated innate immune responses. PLOS One 5(1): e8808.

28. Guillaume van Niel, Richard Wubbolts, Toine Ten Broeke, Sonja I Buschow Ferry A Ossendorp, et al. (2006) Dendritic cells regulate exposure of MHC Class II at their plasma membrane by oligoubiquitination. Immunity 25(6): 885-894.

29. F Alfinito, A Lamberti, M R Capobianchi, M Nigro, G De Rosa, et al. (1994) T cell growth-promoting activity of interferon-gamma. Mitogenic effect of the recombinant cytokine on cells from a human T-chronic lymphocytic leukemia. Leukemia 8(8): 1294-1300.
30. Purnima Bhat, Graham Leggatt, Nigel Waterhouse, Ian H Frazer (2017) Interferon- $\gamma$ derived from cytotoxic lymphocytes directly enhances their motility and cytotoxicity. Cell Death and Disease 8(6): e2836.

31. Yu fei Pan, Li wei Dong, Min Wang, Guang zhen Yang, Jian Zhang, et al. (2013) Signal regulatory protein a negatively regulates mast-cell activation following FceRI aggregation. European Journal of Immunology 43(6): 1598-607.

32. Yoji Murata, Takenori Kotani, Hiroshi Ohnishi, Takashi Matozaki (2014) The CD47-SIRPa signalling system: its physiological roles and therapeutic application. The Journal of Biochemistry 155(6): 335-344.

33. ZI Han Ye, Xiao Ming Jiang, Mu Yang Huang, Yu Lian Xu, Yu Chi Chen, et al. (2021) Regulation of CD47 expression by interferon-gamma in cancer cells. Translational Oncology 14(9).

34. Hyo Jin Kim, Ya Ping Chen, Hongyan Wu, Tammy PT, Jose CV, et al. (2019) SIRPa expression delineates subsets of intratumoral monocyte/ macrophages with different functional and prognostic impact in follicular lymphoma. Blood Cancer Journal 84.

35. Metis Hasipek, Yihong Guan, Dale Grabowski, Xiaorong Gu, Yogenthiran Saunthararajah, et al. (2020) Role of Oligoadenylate Synthetases in Myeloid Neoplasia. Blood 136(1): 29-30.

36. Paulo C Rodriguez, Augusto C Ochoa, Amir A Al-Khami (2017) Arginine metabolism in myeloid cells shapes innate and adaptive immunity. Frontiers in Immunology 8: 93.

37. Jagdip S Jaswal, Wendy Keung, Wei Wang, John R Ussher, Gary D Lopaschuk, et al. (2011) Targeting fatty acid and carbohydrate oxidation-A novel therapeutic intervention in the ischemic and failing heart. Biochimica et Biophysica Acta (BBA)-Molecular Cell Research 1813(7): 1333-1350.

38. Kumari A (2018) Beta Oxidation of Fatty Acids Pages. Sweet Biochemistry p. 17-19.

39. Kumari A (2018) Fatty acid biosynthesis. Sweet Biochemistry p. 21-24.

40. Natasha Fillmore, Osama Abo Alrob, Gary D. Lopaschuk Fatty acid betaoxidation. AOCS Lipid Library.

41. Mingchao Zhang, Weihai Ying (2019) NAD+ deficiency is a common central pathological factor of a number of diseases and aging: mechanisms and therapeutic implications. Antioxidant and Redox Signalling 30(6): 890-905.

42. Hassina Massudi, Ross Grant, Gilles J Guillemin, Nady Braidy (2012) NAD+ metabolism and oxidative stress: the golden nucleotide on a crown of thorns. Redox Report 17(1): 28-46.

43. Eui-Cheol Shin, Federico Perosa, Angelo Vacca, Franco Dammacco, Vito Racanelli, et al. (2013) MHC Class I antigen processing and presenting machinery: organization, function, and defects in tumor cells. JNCI: Journal of the National Cancer Institute 105(16): 1172-1187.

44. R Li, Y Shen, L B Yang, L F Lue, C Finch, et al. (2000) Estrogen enhances uptake of amyloid beta-protein by microglia derived from the human cortex. Journal of Neurochemistry 75(4): 1447-1454

45. Adora A Lin, Sara E Wojciechowski, David A Hildeman (2010) Androgens suppress antigen-specific $\mathrm{T}$ cell responses and IFN $\gamma$ production during intracranial LCMV infection. Journal of Neuroimmunology 226(1-2): $8-19$. 